have an inhibitive effect on the formation of the Bénard convection, as was recently demonstrated by Nakagawa ${ }^{2}$. Chandrasekhar ${ }^{3}$ also points out that with motions at right angles to the magnetic field, the 'closing in' of the lines to produce the usual Bénard cell patterns becomes increasingly difficult. $\mathrm{He}$ also mentions that the lines around sunspots (photographed in hydrogen radiation) may be due to the inhibitive effect of their magnetic fields.

Research Department, W. C. Levengood

Libbey-Owens-Ford Glass Company,

1701 East Broadway,

Toledo 5, Ohio. Oct. 17.

${ }^{1}$ Sci. Amer. 186, No. 5, 26 (1952).

${ }^{2}$ Nature, 175, 118 (1955).

${ }^{3}$ Phil. Mag., 43, 501 (1952).

\section{Relation between the Initial Instantaneous Extension in Creep and the Yield Stress and Coefficient of Work-hardening in Polycrystalline Metals}

IN the course of work on the creep of polycrystalline lead (99.99 per cent) under constant stress in the range $20-105^{\circ} \mathrm{C}$, a full account of which is being prepared, it was found possible to measure the initial instantaneous extension with reasonable accuracy at the lower end of the temperature scale. At higher temperatures the period of low strain-rate, which intervened between the termination of the initial extension and the onset of the transient creep, diminished and the accuracy of measurement suffered in consequence. Nevertheless, as can be seen from Fig. 1, it was possible to establish that the tensile
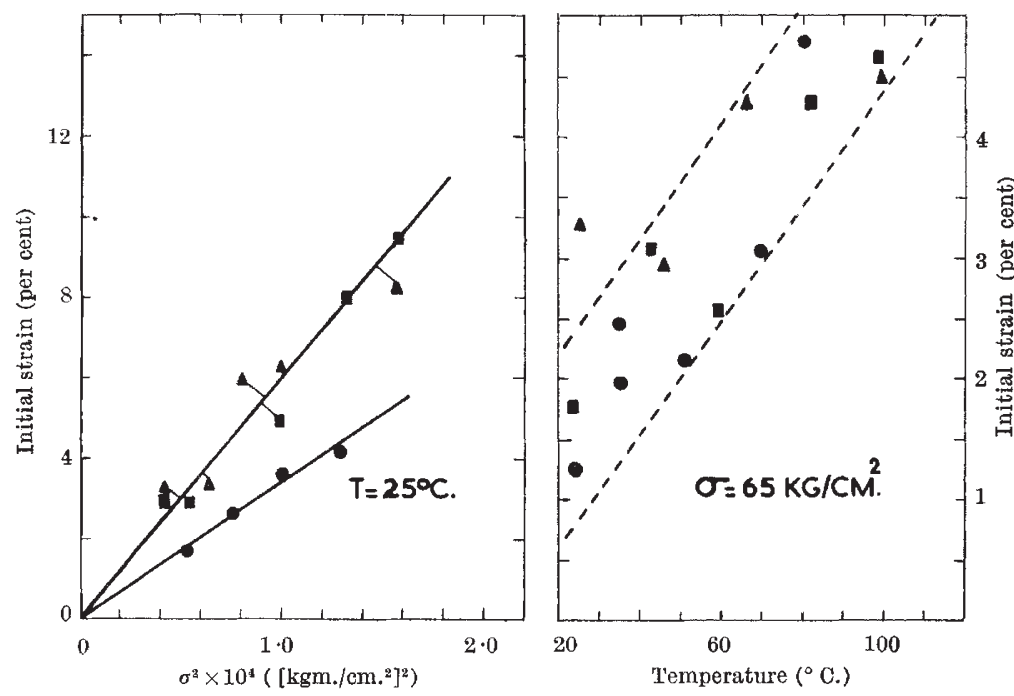

Fig. 1. Mean grain diameter: $0,0.11 \mathrm{~mm}$; $\Delta 0.06 \mathrm{~mm}$; $\mathbf{\omega}, 0.04 \mathrm{~mm}$. of slightly deformed alpha-brass, but particularly the uniformity of the spacing between adjacent pile-ups, suggested that the hardening of the brass was of the interaction type, treated theoretically by Mott ${ }^{2}$, which also leads to a parabolic strain-hardening law. An attempt was therefore made to apply this theory of interaction-hardening to the results obtained in the present work.

If, to conform with the method of testing, the shear stress is replaced by $\frac{1}{2} \sigma$, and the tensile strain is put equal to the shear strain, Mott's theory gives

$$
\chi=G^{2} b n / \pi^{2} L
$$

where $2 L$ is the mean grain diameter, $b$ the interatomic spacing in the slip direction, $G$ the shear modulus and $n$ the number of dislocation in the pile-up. According to Mott,

$$
n \simeq(2 \pi L / G b) \tau_{y}
$$

where $\tau_{y}$ is the yield stress in shear of the undeformed metal. Since, judging from Jacquet's results, $n$ does not appear to be significantly dependent on $L$ for grain-sizes as used by him, and also in the work here discussed, it follows that $\tau_{y}$ should be approximately inversely proportional to the grain-size. Further, from the above relations,

$$
\chi \simeq(2 / \pi) G \tau_{y}
$$

which is independent of $n$. Now, very slow creep in polycrystalline lead is observed at tensile stresses ${ }^{3}$ of about $30 \mathrm{kgm} . / \mathrm{cm} .{ }^{2}$, so that $\tau_{y}$ is less than $15 \mathrm{kgm}$./ $\mathrm{cm}{ }^{2}$ at $25^{\circ} \mathrm{C}$, but must be greater than $3 \mathrm{kgm} . / \mathrm{cm} .{ }^{2}$, which is the yield stress in shear for single crystals of high purity ${ }^{4}$. Therefore, taking $\tau_{y}=10 \mathrm{kgm} . / \mathrm{cm} .^{2}$ and $G=5 \times 10^{4} \mathrm{kgm} . / \mathrm{cm} .^{2}$, the last equation yields $\chi=3.2 \times 10^{5} \mathrm{kgm} . / \mathrm{cm} .{ }^{2}$, which is in satisfactory agreement with the values obtained from the slopes of the lines in the left-hand figure : $2.8 \times 10^{5} \mathrm{kgm} . / \mathrm{cm} .^{2}$ for $2 L=0.11 \mathrm{~mm}$. , and $1.7 \times 10^{5}$ $\mathrm{kgm} . / \mathrm{cm} .^{2}$ for specimens with grain diameters $2 L=0.04$ and $0.06 \mathrm{~mm}$. Owing to poor resolution of the experimental results in the last two cases, separate $\chi$-values were not determined for these grainsizes. It is apparent, however, that the ratio of grain sizes is close to the corresponding ratio of $\chi^{-1}$-values. The value of $n$ which is required to satisfy these results is of the order of 100 at $25^{\circ} \mathrm{C}$., which is comparable with the mean value of the number of dislocations por pile-up as shown in Jacquet's micrographs. The comparatively high temperaturedependence of $\chi$, indicated by the results in Fig. 1 (right), is of about the same magnitude as that of

stress $\sigma$ was related to the initial tensile strain $\varepsilon$ by the work-hardening law characteristic of polycrystalline metals of cubic symmetry :

$$
\sigma^{2} / \varepsilon=\chi(T)
$$

where $\chi(T)$ is the temperature-dependent coefficient of work-hardening.

Jacquet's electron-micrographic evidence ${ }^{1}$ of the formation of dislocation 'pile-ups' at grain boundaries the yield stress in shear of lead single crystals in the same temperature-range ${ }^{4}$.

Department of Metallurgy

University, Leeds, 2. Dec. 5.

${ }^{1}$ Jacquet, P. A., Acta Met., 2, 752 and 770 (1954).

${ }^{2}$ Mott, N. F., Phil. Mag., 43, 1115 (1952); 44, 742 (1953).

${ }^{3}$ Tyte, L. C., Proc. Phys. Soc., 51, 203 (1939).

${ }^{4}$ Neurath, P. W., and Koehler, J. S., J. App. Phys., 18, 79 (1951). 